ISIS 2003 Highlight report

BNL-71897-2003-IR

INFORMAL REPORT

\title{
Spinons in a strongly correlated copper oxide chain
}

\author{
Hyungje Woo (Brookhaven National Laboratory/ISIS) \\ Igor Zaliznyak (Brookhaven National Lab) \\ Toby Perring (ISIS) \\ Collin Broholm (Johns Hopkins University) \\ Chris Frost (ISIS) \\ Hidenori Takagi (University of Tokyo)
}

\begin{abstract}
Experimental realisations of model magnetic systems - ones in which the interactions between the magnetic ions have a particularly simple form - are test-beds for our fundamental understanding of magnetic phenomena. If we are to understand the unusual properties of complex and potentially useful materials in which magnetism plays an important role, such as colossal magnetoresistive oxides or high temperature superconductors, we must also understand the properties of simple systems. $\mathrm{SrCuO}_{2}$ is an example of a one-dimensional magnetic system in which the characteristic energy of excitations from the magnetic ground state is comparable to that of charge motion along the chains. Our results show that the spectrum of magnetic excitations is nevertheless well described by that for the limiting case when charge and spin dynamics are separable.
\end{abstract}

As the dimensionality of a magnetic insulator and as the spin on the magnetic ions is reduced, quantum fluctuations become ever more important in the description of the magnetism. When the interactions are only along a chain, that is, the magnetism is one-dimensional, quantum fluctuations destroy the long-range order that is otherwise expected when there is antiferromagnetic exchange between the ions. The classical picture of magnetic excitations as spin waves, in which the magnetic moments coherently precess about their mean direction, no longer applies. The most extreme case is when the ions have spin $S=1 / 2$, in which case the elementary excitations are spinons - domain walls in the spin system corresponding to inversion of Néel ordering - that can only be created in pairs. As the spinons can independently propagate along the chains with a dispersion relation $\varepsilon=\varepsilon_{L}\left(q^{\text {chain }}\right)$ which relates the energy $\varepsilon$ of the spinon to its momentum $\mathrm{q}^{\text {chain }}$, the resulting spectrum of magnetic excitations $S\left(\mathrm{q}^{\text {chain }}, \varepsilon\right)$ is a continuum that embraces all possible pairs of excitations $\mathrm{q}^{\text {chain }}=\mathrm{q}_{1}{ }^{\text {chain }}+\mathrm{q}_{2}{ }^{\text {chain }}, \varepsilon=\varepsilon_{\mathrm{L}}\left(\mathrm{q}_{1}{ }^{\text {chain }}\right)+\varepsilon_{\mathrm{L}}\left(\mathrm{q}_{2}{ }^{\text {chain }}\right)$. Detailed predictions from theoretical treatments and numerical simulations of the $S=1 / 2$ chain have been thoroughly tested in a number of systems, but always when the strength of the magnetic interactions is more than an order of magnitude smaller than the energy required to excite a charge from one ion to a neighbouring ion. A question that arises is whether or not it still makes sense to treat the spin excitations separately from the charge excitations if the two have comparable energies.

$\mathrm{SrCuO}_{2}$ is a good candidate material in which to study this issue. The copper ions have spin $\mathrm{S}=1 / 2$ and are arranged in pairs of chains along the crystallographic $c$-axis. Neighbouring copper ions interact strongly along chains with the ubiquitous Heisenberg Hamiltonian, $H=J \Sigma_{\mathrm{i}} \mathbf{S}_{\mathrm{i}} \cdot \mathbf{S}_{\mathrm{i}+1}$, but extremely weakly between chains, making the material an almost ideal example of the $S=1 / 2$ Heisenberg antiferromagnetic chain. The spinon excitation spectrum has full width in energy of $\pi J$, which in this material is $\sim 0.6 \mathrm{eV}$ on the basis of estimates of $J$ from magnetisation. This is almost the same as the experimentally measured charge excitation gap of $\Delta_{\mathrm{c}}=0.75 \mathrm{eV}$. Measuring the magnetic excitations to such high energies is a technically challenging task that has only become possible with the highly pixellated detector array on MAPS. Images of 


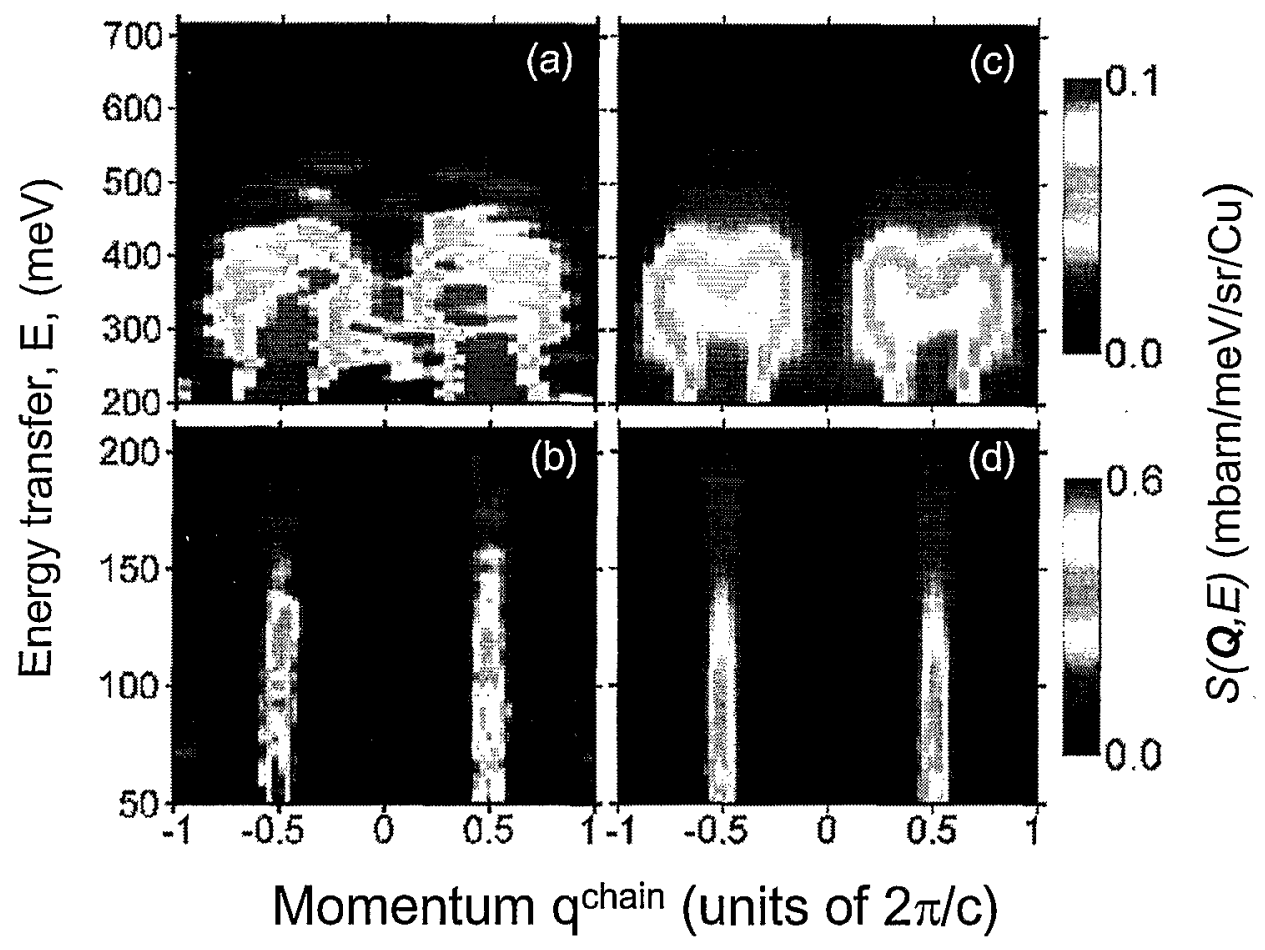

Figure 1: Maps of the scattering intensity in $\mathrm{SrCuO}_{2}$ taken on MAPS with incident neutron energy (a) $848 \mathrm{meV}$ (b) $240 \mathrm{meV}$, with corresponding simulations in (c) and (d).

$S\left(\mathrm{q}^{\text {chain }}, \varepsilon\right)$ from a $3.9 \mathrm{~g}$ single crystal were taken on MAPS with incident neutron energies from $0.1 \mathrm{eV}$ to $1.0 \mathrm{eV}$ in order to study the full spinon spectrum. Figures $1(\mathrm{a})$ and $1(\mathrm{~b})$ show representative examples that focus on the higher and lower energy regions, together with corresponding simulations using the theoretical expression for the continuum scattering with $J=228 \mathrm{meV}$ for the Heisenberg Hamiltonian. The non-magnetic scattering has been subtracted from the data, and the simulations include the effect of the instrument resolution. Qualitatively at least, there is broad agreement between experiment and theory. Figure 2 shows three representative constant- $\varepsilon$ cuts from each of the two images in Figure 1 (before subtraction of the non-magnetic scattering). In each panel, the solid line shows the convolution of the theoretical continuum scattering with the instrumental resolution (together with the momentum independent non-magnetic scattering). As $\varepsilon$ increases, the intensity centred at $q^{\text {chain }}=0.5$ broadens until $\varepsilon=\pi J / 2=360 \mathrm{meV}$, above which it fills the bulk of the Brillouin zone. The continuum is bounded from below by the dispersion for the spinons, $\varepsilon_{\mathrm{L}}\left(\mathrm{q}^{\text {chain }}\right)$, and from above by the same functional form but with twice the energy scale and half the periodicity, as shown by the solid lines in Figure 3. The same bounds are indicated in Figure 2 by dashed lines. The excellent agreement between data and theory in Figure 2, and cuts from intensity maps at other energies, shows that the spinon spectrum for the purely spin Heisenberg Hamiltonian applies even in the case when the width of the spinon continuum, $\pi J$, is the same as the charge gap $\Delta_{c}$. The full set of data is summarised in Figure 3. Independent values for $J$ were obtained from each cut (thus six values from. Figure 2, for example), which were used to place a point on the lower or upper 
bounds to the continuum. The vertical bars show the width of each constant- $\varepsilon$ cut, the horizontal bars indicate the uncertainty in $\mathrm{q}^{\text {chain }}$ arising from the uncertainty in $J$. A single value of $J=228 \pm 10 \mathrm{meV}$ describes all of the data, together with a single intensity pre-scale factor.

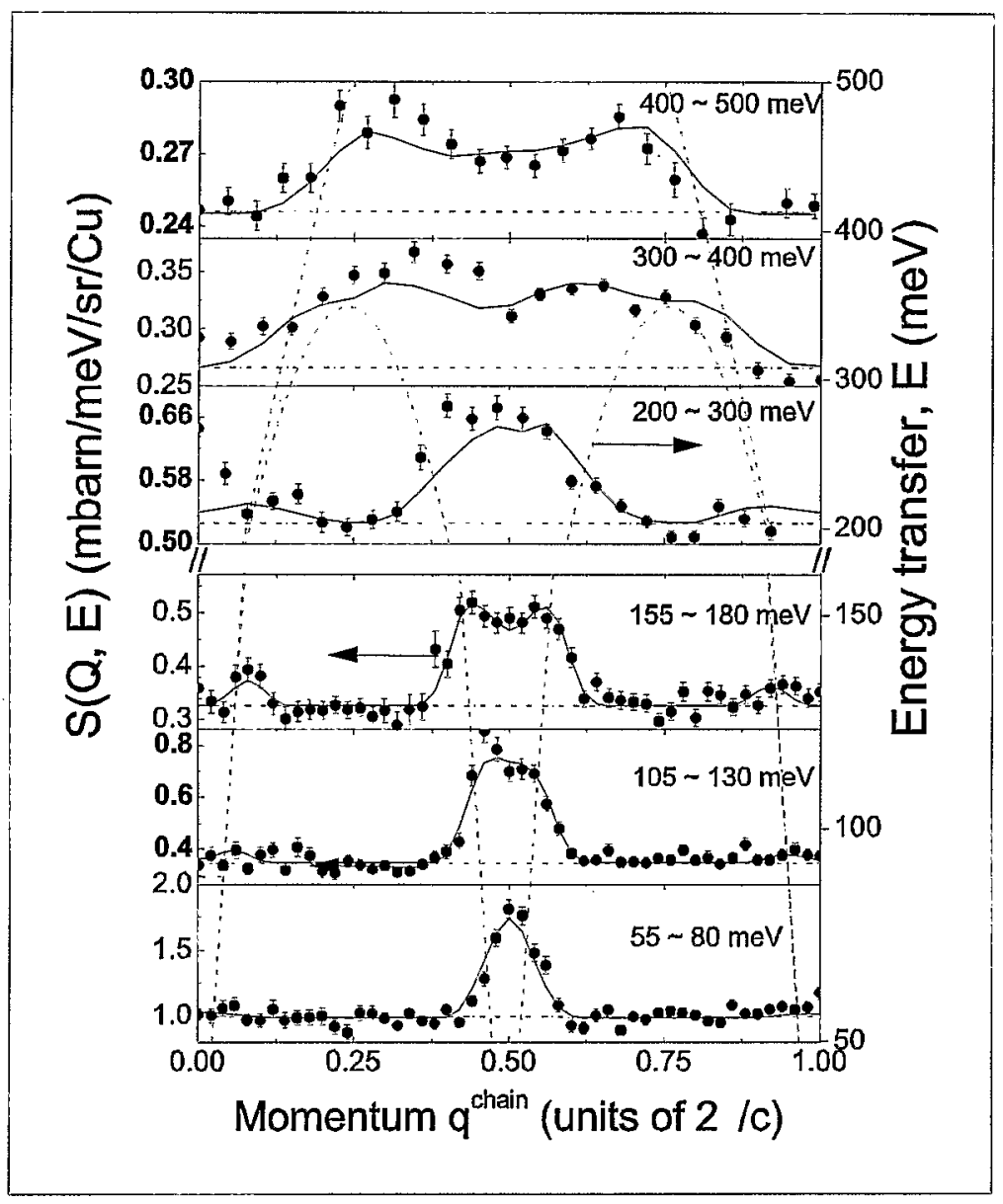

Figure 2: Selected constant- $\varepsilon$ cuts from the data with incident energy $848 \mathrm{meV}$ (upper three panels) and $240 \mathrm{meV}$ (lower three panels). The energy window for each cut is shown in the upper right of each panel. 
ISIS 2003 Highlight report

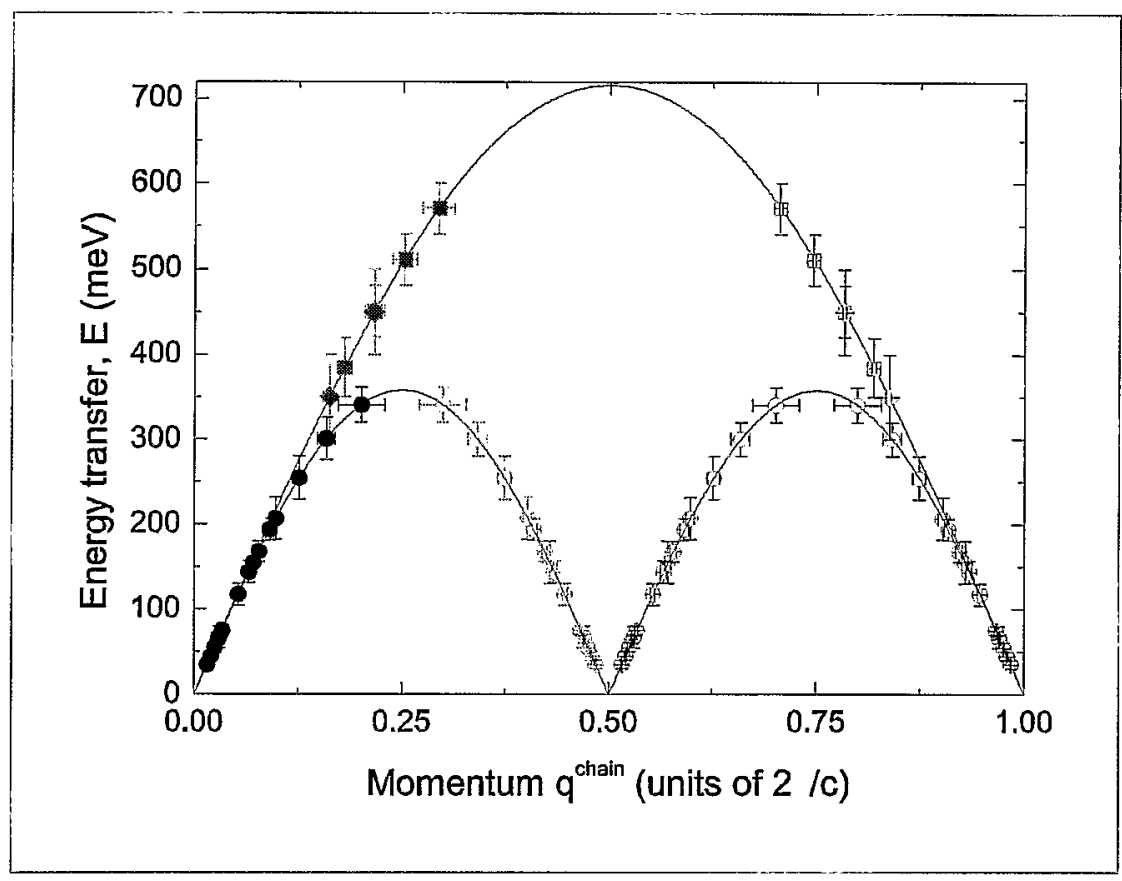

Figure 3: Lower and upper bounds of the spinon continuum scattering. Red markers indicate independent measurements of J. Symmetry equivalent points are marked in black. 